CORRECTION

\title{
Matrix turnover linked to dietary weight loss
}

Dario Ummarino

Nature Reviews Rheumatology 13, 512 (2017)

In the version of this Research Highlight initially published online, serum markers of matrix metalloproteinase-mediated type I collagen degradation were incorrectly said to be higher among patients undergoing diet-induced weight loss, with or without exercise, at 18 months than in patients undertaking exercise only, when these serum markers are actually lower among these patients than in patients undertaking exercise only. This error has been corrected in the online and Print version of the Research Highlight. 